\title{
Résultats préliminaires concernant la mycorhization contrôlée de vitroplants de chêne (Quercus robur $L$.).
}

\author{
J. LEI, J. DEXHEIMER
}

C.N.R.S., Laboratoire de Biologie des ligneux, J.E. 034613, Université de Nancy I, B.P. 239, F 54506 Vandouvre Cedex

\begin{abstract}
Résumé
Les auteurs présentent les résultats préliminaires de la mycorhization contrôlée de vitroplants de chêne. Il a été utilisé quatre souches mycorhizogènes et trois ont formé in vitro des mycorhizes avec les racines des plantules de chêne.

Ces mycorhizes présentent une morphologie et une organisation structurale identiques à celles observées dans les conditions naturelles.

Mots clés : Vitroplants, Quercus robur, ectomycorhizes, Basidiomycètes, mycorhization contrôlée, cytologie.
\end{abstract}

\section{Introduction}

Un nombre croissant d'espèces sont multipliées par les techniques de culture in vitro. En sylviculture, ces techniques présentent un grand intérêt car elles permettent le clonage rapide d'essences présentant des caractéristiques précises (qualité du bois, mode de croissance, aptitude écologique...).

Toutefois, même lorsque tous les problèmes liés à la multiplication végétative in vitro ont été résolus, il reste toujours un point délicat qui est celui du sevrage des vitroplants. La réinstallation en conditions naturelles de plantules élevées en milieu axénique constitue pour ces dernières un traumatisme parfois fatal.

En particulier, les racines vont être brutalement confrontées à la microflore tellurique renfermant, certes des organismes symbiotes, mais aussi de nombreux agents pathogènes. De plus, lorsque la symbiose racinaire s'établit dans les conditions naturelles, elle n'associe pas forcément le partenaire fongique le plus efficient. Il apparaît donc comme tout à fait logique d'essayer de réaliser des mycorhizations contrôlées précoces alors que les vitroplants sont encore en conditions axéniques, ce qui a l'avantage d'équiper les racines du vitroplant d'un complexe symbiotique efficace, lui assurant de meilleures chances de survie.

Cependant, divers problèmes apparaissent avec l'utilisation de ce type de matériel :

- Les racines formées en conditions axéniques, en milieu totalement artificiel, sont-elles aptes à former des mycorhizes et quel sera le comportement du champignon symbiote vis-à-vis de telles racines (non reconnaissance, formation d'une mycorhize ou pathogénicité)? 
- Enfin, s'il s'établit des mycorhizes, présenteront-elles une structure normale, en l'absence de concurrents et en milieu confiné ?

- Ces mycorhizes se maintiennent-elles lors du passage en conditions naturelles?

Pour tenter de répondre à ces questions nous avons essayé de mycorhizer en conditions axéniques des vitroplants de chêne. Le présent article présente les résultats préliminaires de ces expériences.

\section{Matériel et méthodes}

\subsection{Matériel}

\subsection{Les vitroplants}

Les vitroplants de Quercus robur L. obtenus par micropropagation de boutures sont préparés par FAvre \& JunCKer (1986). Ces vitroplants, âgés de 3 mois, présentent un système racinaire bien développé. La tige aérienne porte de 6 à 8 feuilles vertes.

\subsection{Souches fongiques et préparation de l'inoculiam}

Nous avons utilisé quatre champignons ectomycohiziens provenant du Laboratoire de Microbiologie forestière du Centre National de: Recherches forestières de l'INRA à Champenoux. Il s'agit de :

\section{Laccaria laccata \\ Hebeloma crustuliniforme \\ Paxillus involutus \\ Suillus granulatus}

La préparation de l'inoculum a été faite selon la technique mise au point par F. LE TACON (Le TACON et al., 1983). Les souches sont conservées en boîtes de Pétri sur du milieu de Pachlewski gélosé.

Pour préparer l'inoculum, des bocaux de $1500 \mathrm{ml}$ sont remplis d'un mélange de $1 /$ 3 de tourbe et de $2 / 3$ de vermiculite, imprégné avec le milieu de Pachlewski et stérilisé à l'autoclave à $120^{\circ}$ pendant 30 minutes. Ce milieu est ensemencé avec des morceaux d'agar provenant des boîtes de Pétri contenant les souches mycorhiziennes. Les bocaux sont mis à incuber pendant 2 mois et après cette période, la totalité du mélange tourbevermiculite est colonisée par le champignon et constitue "l'inoculum ».

\subsection{Mycorhization contrôlée}

Le milieu de synthèse mycorhizienne renferme un mélange de tourbe-vermiculite et de la solution MMN (MARX, 1969). Les proportions, en volume, sont tourbe : 1, vermiculite : 4, solution MMN : 1,5. Le milieu est stérilisé à $120^{\circ}$ pendant 30 minutes puis une petite quantité d'inoculum (1/10 du volume total) est ajoutée au milieu.

Les vitroplants de chêne, sont prélevés stérilement dans leur tube de culture. Leur système racinaire est lavé soigneusement par l'eau distillée stérilisée pour éliminer toute 
trace de milieu de culture, puis ils sont transplantés sur le mélange tourbe vermiculite inoculé avec le champignon. Les récipients de culture (bocaux de $1000 \mathrm{ml}$ ou tubes de $300 \mathrm{ml}$ ) sont entourés de parafilm et mis dans une chambre de culture avec 10 heures de lumière par jour.

\subsection{Techniques d'observation}

\subsection{Microscopie photonique}

La transparence des parois des récipients de culture permet une observation directe, sous la loupe binoculaire, ou la macrophotographie des mycorhizes.

De plus, des coupes semi-minces sont réalisées à partir des objets préparés pour la microscopie électronique. Les coupes sont recueillies sur une lame, collées par un léger chauffage et colorées par le bleu toluidine à $\mathrm{pH}$ alcalin. L'utilisation de telles coupes nous permet d'obtenir des indications très précises sur l'organisation des mycorhizes.

\subsection{Microscopie électronique}

Pour préciser l'organisation des mycorhizes obtenues, nous avons aussi pratiqué des contrôles en cytologie ultrastructurale.

Les échantillons sont préparés selon les techniques mises au point par DeXHEIMER et al. (1986).

Les racines courtes, entourées par un feutrage mycélien sont fixées dans le

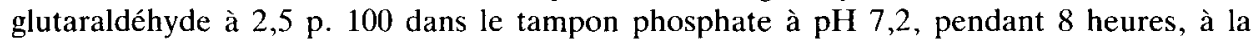
température de la glace fondante. Ils sont ensuite rincés toute une nuit à froid $\left(0^{\circ}\right)$ dans le tampon phosphate, puis ils sont postfixés par le tétroxyde d'osmium à 2 p. 100 dans le même tampon. Enfin, ils sont déshydratés par l'acétone et inclus dans l'épon 812. L’imprégnation par les résines est très lente et dure plus d'un mois.

Les coupes effectuées à l'aide d'un ultramicrotome sont recueillies sur des grilles en cuivre (G 300) et après une double coloration par l'acétate d'uranyle et le citrate de plomb, sont examinées à l'aide des microscopes électroniques Siemens $102 \mathrm{~A}$ et Zeiss EM 9S2.

\section{Résultats}

Après 1 mois, les vitroplants de chêne, repiqués sur le milieu de mycorhization, ont développé un système racinaire important. Certaines racines appliquées sur la paroi de verre des récipients peuvent être observées directement sous la loupe binoculaire.

Pour trois des souches mycorhizogènes testées (Laccaria laccata, Hebeloma crustuliniforme, Paxillus involutus), nous avons observé des racines courtes entourées d'un feutrage mycélien dense.

Pour le Laccaria laccata, la racine courte (1 à $3 \mathrm{~mm}$ de long), entourée du feutrage mycélien, est monodiale, ramifiée pyramidalement. Sa couleur est blanche, légèrement violacée. Les hyphes frangeantes sont abondantes. Les parties âgées présentent un brunissement très net (fig. 1). 

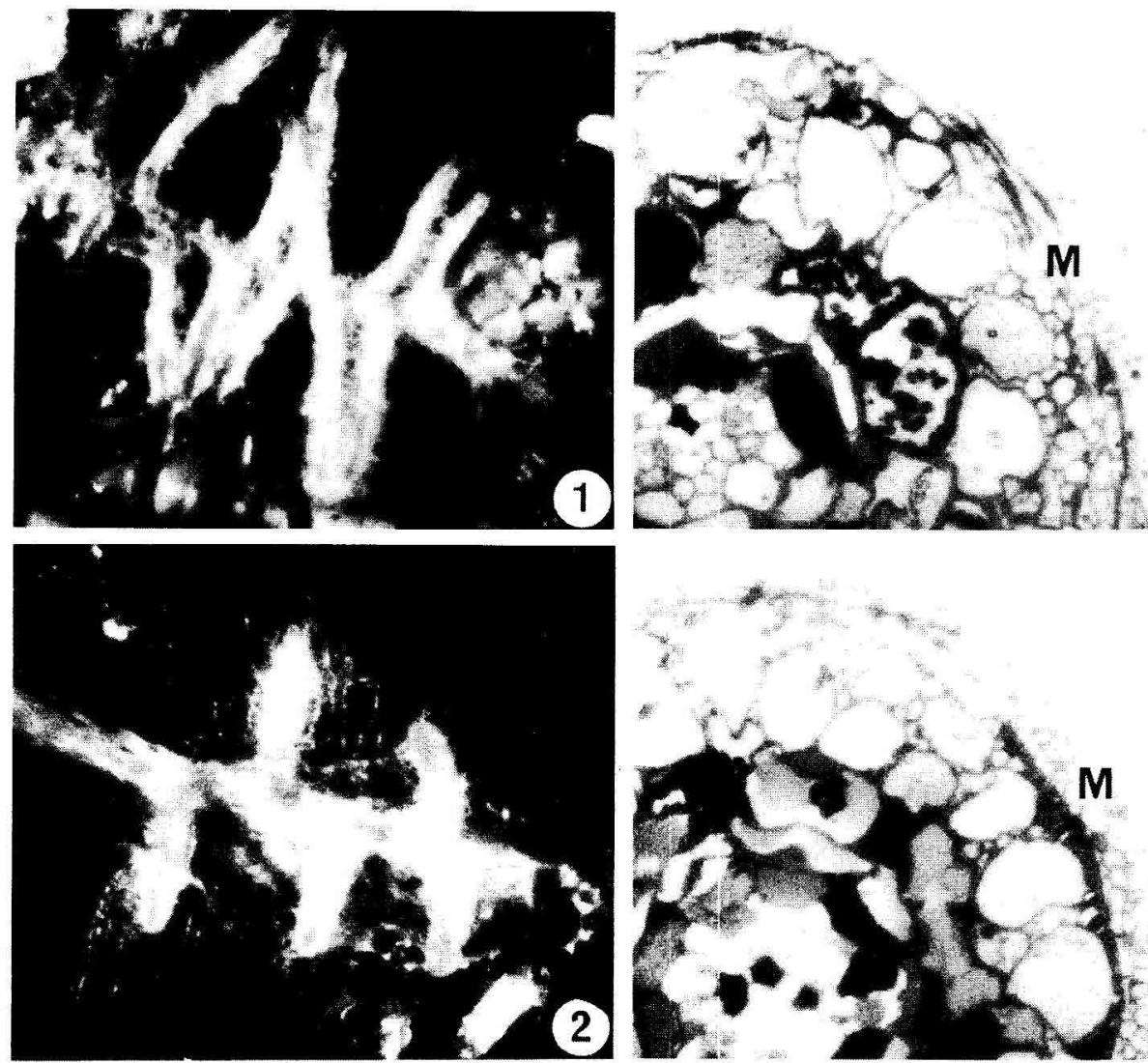

5
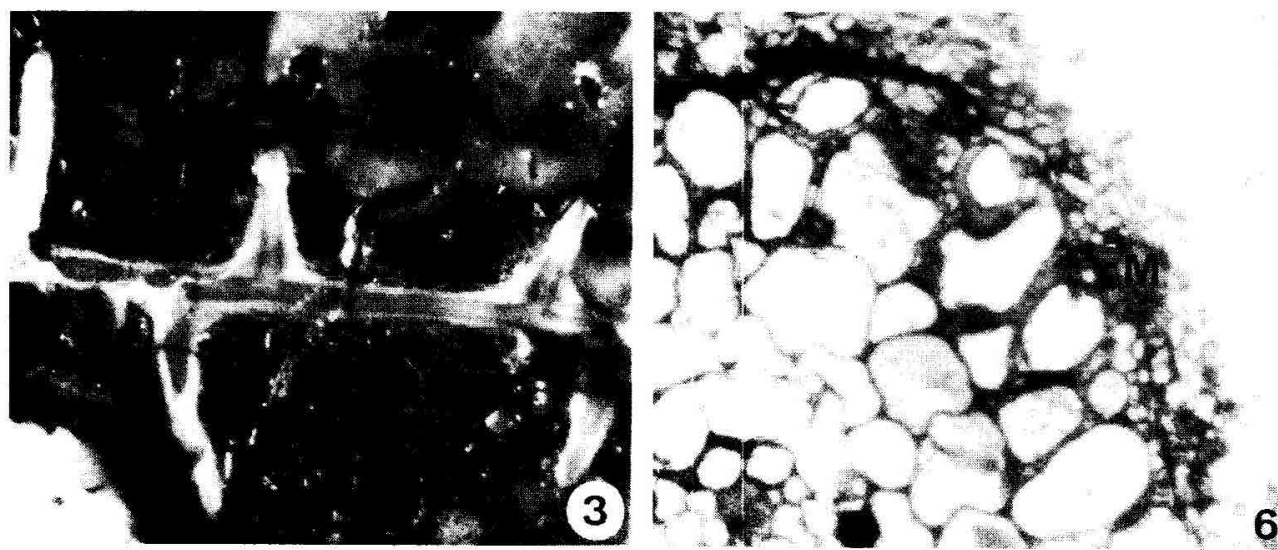

Abréviations: $\mathrm{Cc}$ : cellule corticale; $\mathrm{HR}$ : hyphe du réseau de Hartig; $\mathrm{M}$ : manteau ; $\mathrm{ME}$ : manteau externe ; $\mathrm{MI}$ : manteau interne ; $\mathrm{N}$ : noyau ; $\mathrm{T}$ : tanins. 
Avec les deux autres espèces, les racines courtes, toujours ramifiées monopodialement, ont un aspect moins pyramidal. Avec l'Hebeloma crustuliniforme, elles sont blanches lorsqu'elles sont jeunes puis les parties âgées brunissent. Les hyphes frangeantes sont de couleur beige (fig. 2). Avec le Paxillus involutus, toute la racine apparaît brune plus ou moins foncée. Les hyphes frangeantes sont beiges (fig. 3).

Dans la plupart des cas, le réseau d'hyphes extramatricielles autour des racines est bien développé. Toutefois, lorsque le milieu est très humide (condensation sur les parois des récipients de culture), il est très réduit, voire même inexistant.

La morphologie de ces " racines courtes" observées in vitro est semblable à celle des mycorhizes produites par ces espèces dans les conditions naturelles (GARBAyE \& WILHEM, 1984 ; VOIRY, 1981).

Avec le Suillus granulatus, nous n'avons observé qu'un feutrage mycélien lâche. Dans les conditions naturelles, il n'a jamais été observé de mycorhize avec ce symbiote (MOLINA \& TRAPPE, 1982).

Ces premières observations macroscopiques, semblent donc indiquer qu'il est possible d'obtenir in vitro, une association mycorhizienne entre les racines de vitroplants de chêne et des espèces fongiques, habituellement symbiotes de cette espèce.

Toutefois, pour confirmer la nature mycorhizienne des formations observées, il nous a paru nécessaire de pratiquer des observations structurales en microscopie photonique et électronique.

Pour les observations en microscopie photonique, nous avons utilisé des coupes semi-minces d'objets préparés pour les études en microscopie électronique. Pour les trois espèces de champignon, nous avons observé autour de la racine, un manteau plus ou moins épais et un réseau de Hartig établi entre les cellules des premières couches du cortex (fig. $4,5,6$ ).

Les observations en microscopie électronique précisent ces résultats.

\section{PLANCHE I}

Figs. 1 à 3. - Macrophotographies de mycorhizes de vitroplants de Quercus robur obtenues en conditions axéniques avec trois champignons mycorhizogènes.

Fig. 1. - Laccaria laccata.

Fig. 2. - Hebeloma crustuliniforme.

Fig. 3. - Paxillus involutus.

Figs. 4 à 6. - Coupes semi-minces transversales des mycorhizes de vitroplants de Quercus robur.

Fig. 4. - Laccaria laccata ( $G x$ 675).

Fig. 5. - Hebeloma crustuliniforme (Gx 570).

Fig. 6. - Paxillus involutus (Gx 495).

\section{PLATE I}

Figs. 1 à 3. - Mycorrhizas of Quercus robur vitroplants obtained under aseptic conditions with three mycorrhizal fungi.

Figs. 4 à 6. - Semi-thin cross sections of mycorrhizas in Quercus robur vitroplant roots. 


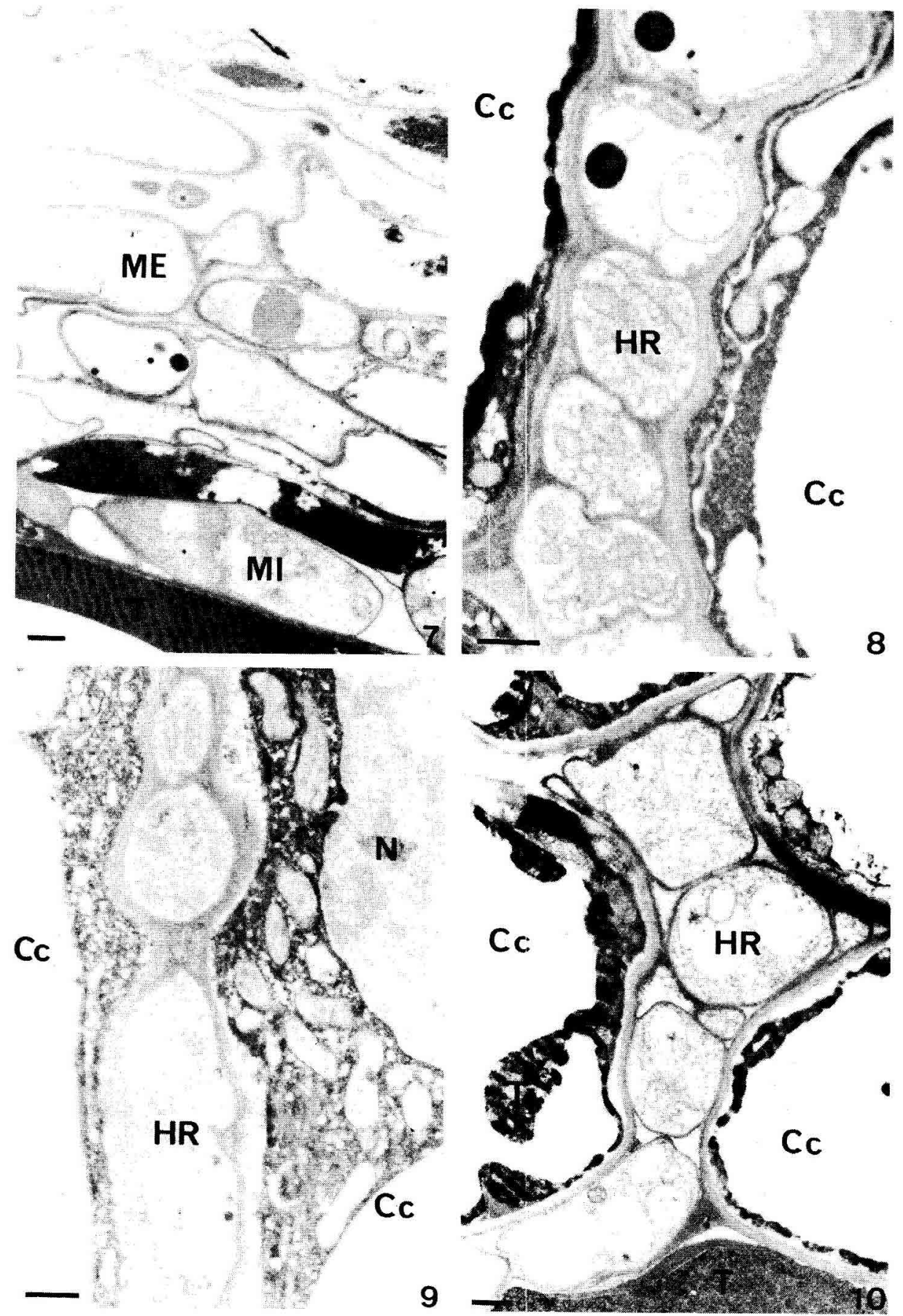


Le manteau apparaît formé de plusieurs épaisseurs d'hyphes noyées dans un ciment interhyphal dont le contraste est plus faible que celui des parois du champignon. Comme dans les mycorhizes observées dans la nature, il est possible de distinguer entre un manteau externe formé d'hyphes vidées de leur contenu et parfois aplaties et déformées et un manteau interne où le cytoplasme des hyphes renferme de nombreux organites (fig. 7).

Des débris denses, sans structure nette sont souvent incorporés au manteau. Ils ont aussi été signalés dans d'autres mycorhizes (DUdDRIDGE \& READ, 1984) et pourraient être des restes, soit de poils absorbants, soit de cellules corticales (fig. 7).

La partie interne du manteau passe progressivement au réseau de Hartig. Celui-ci est unisérié et s'insinue entre les cellules corticales. Les sections d'hyphes montrent qu'elles contiennent un cytoplasme actif avec de nombreux organites et en particulier des mitochondries. Les vacuoles fongiques renferment des inclusions arrondies, denses aux électrons qui ont été interprétées dans d'autres mycorhizes comme correspondant à des granules de polyphosphate (Strullu, 1976 ; Strullu et al., 1980 ; Strullu, 1982) (fig. 8).

Les cellules corticales de l'hôte présentent un mince film cytoplasmique pariétal et une grande vacuole centrale (fig. 9). Certaines montrent des dépôts denses de tanins le long du tonoplaste (fig. 8,10 ). Toutefois, au stade où nous avons pratiqué nos observations, nous avons rarement observé des tanins occupant la totalité de la vacuole, comme cela est souvent décrit dans les ectomycorhizes.

\section{PLANCHE II}

Etude en microscopie électronique des mycorhizes d'Hebeloma crustuliniforme.

FIG. 7. - Coupe transversale du manteau. Le manteau externe est formé par des hyphes présentant un cytoplasme dégénérescent ou totalement disparu. Le manteau interne renferme des hyphes montrant un cytoplasme actif $(G x 5400)$.

FIG. 8. - Réseau de Hartig. Noter la présence de granules denses (polyphosphates) dans les vacuoles fongiques (Gx 8500).

Fı́. 9. - Réseau de Hartig. Les cellules corticales adjacentes au réseau de Hartig présentent une grosse vacuole centrale et un film cytoplasmique pariétal (Gx 7250 ).

Fig. 10. - Réseau de Hartig. Certaines cellules corticales de l'hôte montrent des dépôts denses de tanins le long du tonoplaste ( $G \times 5400$ ).

\section{PLATE II}

Electron microscope study of Oak vitroplant mycorrhizas with Hebeloma crustuliniforme.

FIG. 7. - Sheath cross section. Hyphae are senescent or dead in the outer sheath; The inner sheath contain the hyphae with active cytoplasm. X 5.400.

FIG. 8. - Hartig net. Note presence of the sense granules (polyphosphate) in fungus vacuoles. $X 8.500$.

Fig. 9. - Hartig net. Cortical cells adjacent to the Hartig net contain large central vacuole and peripheral cytoplasm. $X 7.250$.

FIG. 10. - Hartig net. Certain host cortical cells show the dense deposits of tannin along tonoplasts. $X 5.400$. 


\section{Discussion et conclusion}

Les observations macroscopiques, complétées par celles en microscopie photonique et électronique montrent sans ambiguité que trois des espèces fongiques que nous avons utilisées (Laccaria laccata, Hebeloma crustuliniforme, Paxillus involutus) forment des mycorhizes identiques morphologiquement et cytologiquement à celles observées dans les conditions naturelles chez le chêne (Vorry, 1981; Edwards \& Gessner, 1984 ; Garbaye \& Wilhem, 1984).

Avec le Suillus granulatus, qui dans les conditions naturelles ne forme pas de mycorhize avec le chêne (Molina \& TRAPPE, 1.982), nous n'avons pas obtenu de mycorhizes in vitro.

Ces résultats montrent que les racines de vitroplants, maintenus en conditions axéniques sont parfaitement capables d'entrer en symbiose avec des champignons mycorhizogènes. La spécificité de ces associations est conservée puisque seuls les champignons formant des mycorhizes dans les conditions naturelles se sont associés aux racines des vitroplants.

Les contrôles cytologiques ont montré que ces mycorhizes, bien que formées in vitro, dans un milieu confiné présentent une organisation identique à celle observée dans les conditions naturelles. Cette identité n'est probablement pas seulement structurale, mais aussi fonctionnelle. En effet, nous avons souvent observé dans les vacuoles du champignon et particulièrement dans le réseau de Hartig, des granules de polyphosphate pouvant indiquer l'existence de transport de phosphore du substrat vers la mycorhize (WoOLHOUSE, 1975).

Enfin, dans une autre étude (Lel \& Dexheimer, non publié), nous avons montré que la répartition et l'évolution des activités phosphatasiques acides est identique dans une mycorhize de vitroplant obtenue en conditions axéniques (Quercus robur/Paxillus involutus) et une mycorhize obtenue en conditions semi-naturelles (culture en containers Spencer-Lemaire de jeunes Pinus sylvestris mycorhizés par Hebeloma crustuliniforme). Ces faits apportent donc un argument supplémentaire quant à l'identité structurale et fonctionnelle entre les mycorhizes de vitroplants érablies en conditions axéniques et les mycorhizes naturelles.

Actuellement, des études sont en cours pour d'autres activités enzymatiques (ATPases plasmalemmiques, phosphatases neutres, glucose-6-phosphatase) pour vérifier cette similitude fonctionnelle. Si elle était confirmée, la technique de mycorhization contrôlée de vitroplants en conditions axéniques serait complémentaire de la multiplication in vitro et serait susceptible d'accroître les chances de survie au moment du sevrage. De plus, au point de vue théorique, on pourrait disposer de mycorhizes parfaitement contrôlées, pour des études fonctionnelles au niveau cellulaire et subcellulaire.

Reçu le 13 novembre 1986.

Accepté le 18 décembre 1986. 


\section{Summary \\ Preliminary results concerning the controlled mycorrhization of oak (Quercus robur L.) vitroplants}

The authors present preliminary results on the controlled mycorrhization of oak vitroplants. Four mycorrhizogenic strains were used and three formed mycorrhiza with oak plantlets roots in vitro.

These mycorrhizae had the same morphology and organisation as those found under natural conditions.

Key words : Vitroplants, Quercus robur, ectomycorrhizas Basidiomycetes, controled mycorrhization, cytology.

\section{Références bibliographiques}

Dexhfimer J., Aubert-Dufresne M.P., Gerard J., Le Tacon F., Mousain D., 1986. Etude de la localisation ultrastructurale des activités phosphatasiques acides dans deux ectomycorhizes Pinus nigra nigricans/Hebeloma crustuliniforme et Pinus pinaster/Pisolithus tinctorius. Bull. Soc. Bot. Fr., 133, 343-352.

Duddridge J.A., READ D.J., 1984. The development and ultrastructure of ectomycorrhizas. I. Ectomycorrhizal development on pine in the field. New Phytol., 96, 565-573.

Edwardos H.H., Gessner R.V., 1984. Light and transmission electron microscopy of English oak ectomycorrhizal short roots. Can. J. Bot., 62, 1327-1335.

FAvRE J.M., Juncker B., 1986. In vitro growth of buds taken from seedlings and adult plant material in Quercus robur L. Plant cell, tissue and organ culture, 8, 49-60.

Garbaye J., Wilhem M.E., 1984. Influence de la mycorhization acquise en pépinière sur la mycorhization de jeunes plantations de chêne. Acta Oecologica/Oecol. Plant., 5 (19), 151-161.

Lei J.Y., Dexheimer J., 1986. Etude des variations, en fonction de l'âge, de la localisation ultrastructurale des activités phosphatasiques acides dans deux ectomycorhizes à Basidiomycètes ef dans un champignon symbiote non associé. Phytomor phology (à paraître).

Le Tacon F., Jung G., Michelot P., Mugnier M., 1983. Efficacité en pépinière forestière d'un inoculum de champignon ectomycorhizien produit en fermenteur et inclus dans une matrice de polymères. Ann. Sci. For., 40 (2), 165-176.

MaRX D.H., 1969. The influence of ectotrophic mycorrhizal fungi on the resistance of pine roots to pathogenic infection. I. Antagonism of mycorrhizal fungi to root pathogenic fungi and soil bacteria. Phytopathol., 59, 153-163.

Molina R., Trappe J.M., 1982. Patterns of ectomycorrhizal host specificity and potential among Pacific Northwest Conifers and fungi. Forest Sci., 28, 423-458.

Strullu D.G., 1976. Etude des relations nutrition-développement et cytologie des mycorhizes chez le Douglas (Pseudotsuga menziesii Mirb.) et les Abiétinées. Thèse d'Etat, Université de Rennes, 1-291.

Strullu D.G., 1982. I. L'Association mycorhizienne. Académie d'Agriculture de France. Extrait du Procès verbal de la séance du 17 février 1982, 344-360.

Strullu D.G., Gourret J.P., 1980. Données ultrastructurales sur l'intégration cellulaire de quelques parasites ou symbiotes de plantes. II. Champignons mycorhiziens. Bull. Soc. Bot. Fr., Actua. Bot., 127 (1), 97-106.

VoIRY H., 1981. Classification morphologique des ectomycorhizes du chêne et du hêtre dans le nord-est de la France. Sonderdruck aus European Journal of Forest Pathology, 11, $284-299$.

Woolmouse H.N., 1975. Membrane structure and transport problems considered in relation to phosphorus and carbohydrate movements and the regulation of endotrophic mycorrhizal associations. In "Endomycorrhizas", Eds Sanders F.E.T., Mosse B., Tinker P.B., Academic Press, Londres, New York, 209-240. 